\title{
Are random controlled trials appropriate for orthodontics?
}

\section{John Mew}

\author{
Clinical Director \\ The London School of Facial Orthodontics
}

\begin{abstract}
In orthodontics, cost effectiveness is especially difficult to assess because of the wide range of problems and cures and a lack of precise scientific information about their success rates. While many would accept that the basic problem is skeletal disproportion, there are diverse views about the underlying cause. Over the last hundred years treatment has ranged from extracting teeth in every patient to never extracting teeth, each view being held with fierce conviction. You would think that a bit of clear-minded research would have settled the debate. But no, there is still wide disagreement.
\end{abstract}

Evidence-Based Dentistry (2002) 3, 35-36. doi:10.1038/sj.ebd.6400099

There is currently some concern about the evaluation of clinical treatment. For instance, if one treatment is twice as good but takes three times as long, is that good or bad? If, more critically, it is twice as good but costs three times as much, do we say yes or no? We talk of clinical effectiveness but how accurate is our assessment? Clinical governance seems here to stay but just how different is this from clinical expedience? If a brain surgeon developed a technique that was four times as costly but twice as beneficial, would the world beat a path to his or her door?

In orthodontics cost effectiveness is especially difficult to assess because of the wide range of problems and cures, and a lack of precise scientific information about their success rates. While many would accept that the basic problem is skeletal disproportion, there are diverse views about the underlying cause. The majority still feel that this is genetic and so concentrate on aligning the teeth mechanically using surgery if the discrepancy is too large. However a substantial minority appear equally certain that the cause is environmental and use functional appliances to try and influence growth. Over the last hundred years treatment has ranged from extracting teeth in every patient to never extracting teeth, each view being held with fierce conviction. You would think that a bit of clear-minded research would have settled the debate. But no, there is still wide disagreement.

The main problem is that it takes two or more years to correct a malocclusion and another ten to twenty to assess the long-term success. Not only do clinicians retire but patients disappear. Most orthodontic studies have been short term and retrospective and David Sackett, former Professor of Evidencedbased Medicine at Oxford, stated in 1985 "Orthodontics is behind such treatment modalities as acupuncture, hypnosis, homeopathy, and orthomolecular therapy and on a par with scientology" ${ }^{1}$. Although he was pleased to see that things had improved by 1994, his colleague Derek Richards, Director of the Centre for Evidencedbased Dentistry was still cautioning
"The current focus of dental schools leans toward the teaching of technical skills rather than scientific thinking" ${ }^{2}$ and Professor Bill Shaw writing in a recent issue of Evidence-Based Dentistry says of orthodontics "Sadly it is hard to see this situation change unless the inadequacy of current knowledge is acknowledged by its practitioners" 3 .

It is obvious that orthodontics desperately needs some sound facts on which treatment can be based. randomized clinical trials (RCTs) appear to be the answer but are they appropriate for orthodontics? Possibly not for several reasons.

1. 'Blinding' is rarely possible.

2. Suitable controls are a basic requirement for almost all medical research. Unfortunately individuals with 32 perfectly straight teeth are rare in civilized societies and there is thus a risk of our accepting the mild malocclusions that we see around us as 'normal'. The best control samples we have are from the Bolton, Burlington and Kings College studies, the occlusions of which are 'good' rather than 'excellent', and available to us only as records. It is interesting to note that these groups tend to have less variation than average population standards. We are at risk of comparing one group of 'abnormals' with another, both of whom have large variations. It is difficult to obtain meaningful results if a sample is 
skewed especially if both the patients and controls are towards the same end of the range of variables.

3. Because of the substantial variations between individual malocclusions, large numbers of patients are necessary to gain 'significant' results. This requires many operators who inevitably have varying clinical experience. Unfortunately the specific skills of the clinician often make more difference than the treatment being tested but this is rarely allowed for.

4. If patients in a trial are distributed randomly, some may be cared for by clinicians for whom the selected treatment might not have been the first choice. This not only raises ethical problems but is of particular relevance to the success of the treatment, which can be highly dependent on the clinician's conviction and enthusiasm.

5. Unfortunately the current emphasis on teaching fixed appliances means that there are now fewer clinicians with wide experience in functional appliances, especially in the schools where most RCTs are being done. In a recent very extensive UK study ${ }^{4}$, some of the clinicians were not only unfamiliar with the appliances being tested, but in at least one instance had never used them before.

6. RCTs are not very suitable for assessing several variables simultaneously, and yet by reducing the number of variables the results can sometimes be prejudiced. For instance a recent RCT in the USA did not expand the maxilla before fitting a Bionator ${ }^{5}$. Thus the occlusion was not 'unlocked' and those familiar with functional appliances would not be surprised to hear that this study failed to show much difference between Headgear, Bionators and controls.

7. However the most important flaw with all research involving functional appliances is that success is almost entirely dependent on the co-operation of the patient and this is almost impossible to ascertain.

8. Sadly most RCTs in orthodontics have been very expensive and because of problems such as those mentioned above, have tended to produce rather negative results. It is therefore likely that funding them may be difficult in future.

Faced with these difficulties where do we go for the answers that orthodontists need so badly? The ideal would be identical twins treated by different techniques by skilled clinicians who were convinced their methods were correct. However it would be very difficult to assemble the material and would undoubtedly present ethical problems.

A viable alternative must remain the Prospective Consecutive Trials (PCTs). In 1971 the British Association of Orthodontists authorized this author to set up such a study. It was designed similarly to Shaw's 'Six Centres Study' that changed cleft palate surgery so dramatically twenty years later. The protocol was for ten different centers each to treat 20 cases with five different specified types of malocclusion (ie 4 class I, 4 class II/ 1 - two of them vertical growers, 3 class II/2, 2 class III, 2 open bite, and 2 deep bite). They were free to choose the severity of the cases themselves but clearly it would have been in their own interests to demonstrate some difficult cases. Unfortunately clinical weaknesses in these circumstances become obvious which may be why no centers were willing to commit themselves at that time, had they done so, we might by now have a better idea of which methods are most effective.

One of the principal advantages of PCTs is that the costs are minimal, requiring little more than duplication of the records so that they can be lodged at the monitoring unit. Some centres see the element of competition as undesirable however this does ensure that skilled operators are used. With suitable reward most patients can be encouraged to return out of retention but those that fail to do so must not be excluded from the results. It does seem that PCTs might provide orthodontics with some of the answers so badly needed.

1. Sackett D. Professor of Evidenced Based Research at Oxord. 1994 'Nine years later; a commentary on revisiting the Moyers symposium'. Craniofacial Growth Series, Center for Human Growth and Development, University of Michigan, Ann Arbor.

2. Richards D. 'London Evidence-based Symposium'. EBD 2000; 2:3-4.

3. Shaw WC. How relevant is the evidencebased process to orthodontics? EBD 2000; 2:7-8.

4. Robinson S. 2001 British Orthodontic Society Annual Conference, Brighton U.K.

5. Tulloch, J.F.C., Phillips, C., and Profitt, W.R. 1998 "Benefit of early Class II treatment: Progress report of a two-phase randomized clinical trial". American Journal of Orthodontics and Dentofacial Orthopedics. Volume 113:62-72.

6. Shaw WC, Asher-McDade A, Brallstrom V, Dahl E, McWilliam J, Molsted K, Plint DA, Prahl-Andersen B, Semb G. A six centre international study of treatment outcomes in patients with cleft lip and palate: part 1 principals and study design. Cleft PalateCran J. 1992; 29:393-397. 